\title{
Allocating Service Periods to Minimize Delay Time*
}

\author{
W. A. Horn \\ Institute for Basic Standards, National Bureau of Standards, \\ Washington, D.C. 20234
}

(May 28, 1968)

\begin{abstract}
Consider a facility which must divide its services, during the time interval $[0, T]$, among $N$ streams of arrivals. The problem treated is that of finding a pattern of service which minimizes total delay to the members of the streams, taking into account the "dead time" which begins each service period. For each stream, it is required that final queue size equal initial size, and that the queue be empty sometime in $[0, T]$. Conditions for feasibility of solutions are given in the case where the instantaneous service rates are bounded above by known constants. In the event that all streams have constant arrival rates and are to be served the same number of times, an optimal service pattern is derived using a recent result of $\mathrm{R}$. Rangarajan and R. M. Oliver.
\end{abstract}

Key Words: Allocation; queueing theory; scheduling; switching theory; traffic flow; transportation theory.

\section{Problem Statement}

This paper presents a further investigation into the type of problem studied in a recent paper by Rangarajan and Oliver. ${ }^{1}$ The problem pertains to the allocation of servicing times among several incoming streams which require "processing" of some kind by a single "server" capable of handling only one stream at a time. The server might for example be a switching point or a congestion point (e.g., a tunnel entrance) in a transport network, in which case "serving" a stream simply means permitting passage to its flow. Or, the server might be a computer handling reservations being arranged at several points, or exercising control on a real-time basis over operations along several links.

The time period during which servicing occurs is assumed to be $[0, T]$, where $T>0$. The rate at which "customers" arrive at stream $i$ is assumed to be the known continuous function $a_{i}(t)$, while the outflow or service-rate function $s_{i}(t)$, which will be defined below, is bounded above by the constant $C_{i}>0$, the capacity when servicing stream $i$. It is further assumed that $a_{i}(t)$ crosses the level $C_{i}$ at most a finite number of times in $[0, T]$. That is, $a_{i}^{-1}\left(C_{i}\right)$ is a set with a finite number of connected components.

The size of the waiting queue in stream $i$ at time $t$ is designated by $Q_{i}(t)$, and we let $q_{i}=Q_{i}(0)$, where it is assumed that each $q_{i}$ is nonnegative. Then clearly

$$
Q_{i}(t)=q_{i}+\int_{0}^{t}\left(a_{i}(\tau)-s_{i}(\tau)\right) d \tau
$$

A cumulative waiting time function $W_{i}(t)$ is defined for each $i$ by

$$
W_{i}(t)=\int_{0}^{t} Q_{i}(\tau) d \tau
$$

\footnotetext{
*Supported by Northeast Corridor Transportation Project, Department of Transportation. No official endorsement is implied.

' R. Rangarajan and R. M. Oliver, Allocations of servicing periods that minimize average delay for $N$ time-shared traffic streams, Transportation Science 1 . $74-80$ (1967).
} 
and a total waiting time function $W(t)$ is defined by

$$
W(t)=\sum_{i=1}^{N} W_{i}(t)
$$

where $N$ is the number of streams.

Since $s_{i}(t)$ will depend on the times at which servicing actually occurs, it is clear that $W_{i}$ and $W$ also depend on these variables. Furthermore, $W_{i}$ and $W$ depend on the quantities $q_{i}$. These functional dependencies may be expressed explicitly when convenient.

Next we define $s_{i}(t)$. As a preliminary, we further limit the generality of the problem by assuming that a given stream $i$ is serviced, within $[0, T]$, only during a finite set of closed intervals $\left\{\left[x_{i j}, y_{i j}\right]\right\}_{j=1}^{m(i)}$ where $m(i)$ is the total number of such intervals of service to stream $i$. No two intervals of servicing, for any streams, may overlap except at their endpoints, and the set of all service intervals for all streams covers $[0, T]$. (The endpoints of these intervals comprise the switching pattern.) Furthermore, for some set of positive constants $\left\{d_{i}\right\}$ called the "dead" times, it is assumed that $x_{i j}+d_{i} \leqslant y_{i j}$, for $i=1,2, \ldots ., N$, and $j=1,2, \ldots, m(i)$.

If stream $i$ is not being serviced at time $t$, then $s_{i}(t)$ is defined to be 0 . If $t$ lies in the service interval $\left[x_{i j}, y_{i j}\right]$, then $s_{i}(t)$ is defined by

$$
s_{i}(t)=\left\{\begin{array}{l}
0: t \epsilon\left[x_{i j}, x_{i j}+d_{i}\right] \\
C_{i}: t \epsilon\left(x_{i j}+d_{i}, y_{i j}\right] \text { and } Q_{i}(t)>0, \\
\min \left(C_{i}, a_{i}(t)\right): t \epsilon\left(x_{i j}+d_{i}, y_{i j}\right] \text { and } Q_{i}(t) \leqslant 0
\end{array}\right.
$$

Now it is clear from (1) that $Q_{i}$ depends on $s_{i}$, whereas the above definition states that $s_{i}$ depends on $Q_{i}$, at least on the intervals $\left(x_{i j}+d_{i}, y_{i j}\right)$. Thus it is necessary to show that there exist unique functions $s_{i}$ and $Q_{i}$ which satisfy (1) and the equation for $s_{i}$. This will be detailed in appendix A.

The above definition of $s_{i}$, together with the fact that $q_{i} \geqslant 0$, assures that $Q_{i}(t) \geqslant 0$ for all $t \epsilon[0, T]$, since if $Q_{i}(t)<0$ for some $t$ then there would exist $t^{\prime}<t$ such that $Q_{i}\left(t^{\prime}\right)=0$, by the continuity of $Q_{i}$. Let $t_{0}^{\prime}$ be the greatest such $t^{\prime}$. Then $Q_{i}(r) \leqslant 0$ for all $r$ such that $t_{0}^{\prime} \leqslant r \leqslant t$, and so $a_{i}(r)-s_{i}(r) \geqslant 0$ in this interval, by the definition of $s_{i}$. This contradicts the fact that

$$
0>Q_{i}(t)=Q_{i}\left(t_{0}^{\prime}\right)+\int_{t_{0}^{\prime}}^{t}\left(a_{i}(r)-s_{i}(r)\right) d r .
$$

The general problem to be considered is that of finding a finite set of intervals $\left[x_{i j}, y_{i j}\right]$ of service, as defined and restricted above, such that the total waiting time $W(T)$ is a minimum, given the initial values $q_{i}$.

We will consider a variant of the general problem in this paper by introducing the restrictions (for $i=1,2, \ldots, N$ )

$$
\begin{gathered}
q_{i}=Q_{i}(0)=Q_{i}(T), \\
Q_{i}(t)=0 \text { for some } t \epsilon[0, T],
\end{gathered}
$$

and by allowing the minimization not only over the intervals of servicing but also over the parameters $q_{i}$ as well. That is, the $q_{i}$ will not be considered fixed but variable, subject to (2) and (3). Constraints (2) and (3) are called the feasibility constraints, and a solution is feasible if it satisfies them.

Imposing constraint (2) has the property of making the problem periodic, in the following sense. If we suppose that $a_{i}$ is defined for all $t \geqslant 0$, rather than for $t \epsilon[0, T]$, with $a_{i}(t+T)=a_{i}(t)$, and that the switching pattern is extended in such a way that stream $i$ is being serviced at time $t+T$ if and only if it is also being serviced at time $t$, then $Q_{i}(0)=Q_{i}(T)$ implies

$$
Q_{i}(t+T)=Q_{i}(t)
$$


It is also seen from this that

$$
W_{i}(T+t)-W_{i}(t)=W_{i}(T)
$$

or,

$$
W_{i}(n T+\theta)=n W_{i}(T)+W_{i}(\theta) .
$$

This in turn implies that

$$
W(t) / t \rightarrow W(T) / T \text { as } t \rightarrow \infty,
$$

so that, in a sense, minimizing $W(T)$ and thus $W(T) / T$ is equivalent to minimizing the "long-term average delay." The motivation for this restriction is that the applications we have in mind refer to ongoing systems rather than isolated occurrences. It seems of little practical use (except for emergency evacuation operations and the like) to formulate the problem as if what happened after time $T$ were of no concern. Condition (2), at least for periodic arrival patterns, implies stability in the sense of "repeatability" for the situation, in particular ruling out unbounded growth of queues over the long run. It might prove worthwhile to investigate the problem variant in which (2) is replaced by

$$
Q_{i}(T) \leqslant Q_{i}(0)
$$

but this version will not be studied here.

\section{Feasibility and Consistency Conditions}

So far it is not known whether there exist feasible solutions of the problem of section 1. This section will be devoted to finding necessary and sufficient conditions for the existence of such solutions.

Since we are considering the problem where not only the points of switching are allowed to vary, but also the $q_{i}$, the first question which arises is how much freedom the $q_{i}$ have, for a given switching pattern, so that conditions (2) and (3) may still be satisfied. The following two lemmas answer this.

LEMma 1. Let $\mathrm{Q}_{\mathrm{i}}$ and $\mathrm{Q}_{\mathrm{i}}^{\prime}$ be the queue-size functions associated with the initial values $\mathrm{q}_{\mathrm{i}}$ and $\mathrm{q}_{\mathrm{i}}^{\prime}$, respectively, and having the same switching pattern. Then $\left|\mathrm{Q}_{\mathrm{i}}(\mathrm{t})-\mathrm{Q}_{\mathrm{i}}^{\prime}(\mathrm{t})\right|$ is a nonincreasing function of $\mathrm{t}$.

Proof. First, $Q_{i}-Q_{i}^{\prime}$ does not change sign. For if $Q_{i}(a)-Q_{i}^{\prime}(a)>0$ and $Q_{i}(b)-Q_{i}^{\prime}(b)<0$, where $a<b$, then by continuity $Q_{i}(t)=Q_{i}^{\prime}(t)$ for some $t \epsilon(a, b)$. But then $Q_{i}(\tau)=Q_{i}^{\prime}(\tau)$ for all $\tau \geqslant t$, contradicting $Q_{i}(b)<Q_{i}^{\prime}(b)$.

Now suppose (say) $Q_{i}(0)>Q_{i}^{\prime}(0)$. Then $Q_{i}(t)-Q_{i}^{\prime}(t)=\left|Q_{i}(t)-Q^{\prime}(t)\right|$, by the above. But $Q_{i}(t)-Q_{i}^{\prime}(t)=q_{i}-q_{i}^{\prime}+\int_{0}^{t}\left(s_{i}^{\prime}(\tau)-s_{i}(\tau)\right) d \tau$, and since $s_{i}^{\prime}(\tau) \leqslant s_{i}(\tau)$ for all $\tau \epsilon[0, T]$ because $Q_{i} \geqslant Q_{i}^{\prime}$, we have that $\left|Q_{i}(t)-Q_{i}^{\prime}(t)\right|$ is nonincreasing. Similarly if $Q_{i}(0)<Q_{i}^{\prime}(0)$ or $Q_{i}(0)=Q_{i}^{\prime}(0)$. LemMa 2. Let $\mathrm{Q}_{\mathrm{i}}$ and $\mathrm{Q}_{\mathrm{i}}^{\prime}$ be two queue-size functions associated with the same switching pattern during $[0, \mathrm{~T}]$ and both satisfying (2). Then $\mathrm{Q}_{\mathrm{i}}$ and $\mathrm{Q}_{\mathrm{i}}^{\prime}$ differ by a constant. Hence there exists at most one feasible solution for each switching pattern.

Proof. From lemma 1,

$$
\left|Q_{i}(0)-Q_{i}^{\prime}(0)\right| \geqslant\left|Q_{i}(t)-Q_{i}^{\prime}(t)\right| \geqslant\left|Q_{i}(T)-Q_{i}^{\prime}(T)\right|
$$


for all $t \epsilon[0, T]$. But by $(2),\left|Q_{i}(0)-Q_{i}^{\prime}(0)\right|=Q_{i}(T)-Q_{i}^{\prime}(T) \mid$. Hence, by the continuity of the queuesize functions, $Q_{i}(t)-Q_{i}^{\prime}(t)=q_{i}-q_{i}^{\prime}$, a constant.

Now if two solutions having the same switching pattern are both feasible, then they satisfy (3) in addition to (2). But if $q_{i}>q_{i}^{\prime}$, for example, then $Q_{i}(t)>Q_{i}^{\prime}(t) \geqslant 0$, by the above, and $Q_{i}$ is not feasible. Therefore $q_{i}=q_{i}^{\prime}$ and so $Q_{i}=Q_{i}^{\prime}$.

The next question to be treated is which patterns of switching times admit feasible solutions. THEOREM 3. Suppose that stream $\mathrm{i}$ is serviced during successive intervals of length

$$
\mathrm{L}_{\mathrm{i} 1}, \mathrm{~L}_{\mathrm{i} 2}, . . ., \mathrm{L}_{\mathrm{im}(\mathrm{i})}
$$

and let $\mathrm{d}_{\mathrm{i}}$ be the dead time for stream $\mathrm{i}$. Then a necessary and sufficient condition that a set of $\mathrm{q}_{\mathrm{i}}$ exist for which the given switching pattern produces a feasible solution is

$$
\mathrm{C}_{\mathrm{i}} \sum_{\mathrm{j}=1}^{\mathrm{m}(\mathrm{i})} \mathrm{L}_{\mathrm{ij}} \geqslant \int_{0}^{\mathrm{T}} \mathrm{a}_{\mathrm{i}}(\mathrm{t}) \mathrm{dt}+\mathrm{m}(\mathrm{i}) \mathrm{C}_{\mathrm{i}} \mathrm{d}_{\mathrm{i}}
$$

for all i.

Proof. Let the above inequality be satisfied. It is clear that, when $q_{i}$ is sufficiently large, $Q_{i}(t)>0$ for all $t \epsilon[0, T]$. Then, by definition, $s_{i}(t)=0$ during nonservice intervals and during a part $d_{i}$ of each service interval, and $s_{i}(t)=C_{i}$ otherwise. Thus for such $q_{i}$,

$$
\int_{0}^{T} s_{i}(t) d t=C_{i} \sum_{j=1}^{m(i)}\left(L_{i j}-d_{i}\right)=C_{i} \sum_{j=1}^{m(i)} L_{i j}-C_{i} m(i) d_{i} \geqslant \int_{0}^{T} a_{i}(t) d t,
$$

so that

$$
Q_{i}(T)=q_{i}+\int_{0}^{T}\left(a_{i}(t)-s_{i}(t)\right) d t \leqslant q_{i}
$$

On the other hand, for $q_{i}=0$ it is clear that $Q_{i}(T) \geqslant q_{i}$. But for a fixed switching pattern, $Q_{i}(T)$ is a continuous function of $q_{i}$. (In fact, by lemma $1,\left|Q_{i}(T)-Q_{i}^{\prime}(T)\right| \leqslant\left|q_{i}-q_{i}^{\prime}\right|$.) Thus there exists a $q_{i}$ for which $Q_{i}(T)=q_{i}$.

Let $q_{i}^{0}$ be the infimum of all such $q_{i}$ satisfying $q_{i}=Q_{i}(T)$ for the given pattern of switching. Let

$$
b=\inf \left\{Q_{i}(t): t \epsilon[0, t], Q_{i}(0)=q_{i}^{o}\right\} .
$$

If $b=0$, then $q_{i}^{0}$ gives a feasible solution. But if $b>0$, then usirig the value $q_{i}=q_{i}^{0}-b / 2$ as the initial queue size, we find that $Q_{i}(t) \geqslant b-b / 2=b / 2$ for all $t \epsilon[0, T]$, by lemma 1 . Thus by the definition of $s_{i}$ we have the same value of $s_{i}(t)$, for each $t \epsilon[0, T]$, for the two initial values $q_{i}^{0}$ and $q_{i}^{0}-b / 2$. But this implies that the solution with initial value $q_{i}^{0}-b / 2$ also satisfies (2), contradicting the definition of $q_{i}^{0}$.

This proves sufficiency. Necessity follows from (2), noting that

$$
\int_{0}^{T} a_{i}(t) d t=\int_{0}^{T} s_{i}(t) d t \leqslant C_{i} \sum_{j=1}^{m(i)}\left(L_{i j}-d_{i}\right) .
$$

Lemma 4. Suppose there exist numbers $\mathrm{T}_{\mathrm{i}}>0$ such that

$$
\sum_{i=1}^{N} T_{i}=T
$$

and

$$
\mathrm{C}_{\mathrm{i}} \mathrm{T}_{\mathrm{i}} \geqslant \int_{0}^{\mathrm{T}} \mathrm{a}_{\mathrm{i}}(\mathrm{t}) \mathrm{dt}+\mathrm{m}(\mathrm{i}) \mathrm{C}_{\mathrm{i}} \mathrm{d}_{\mathrm{i}}
$$


Then there exists a feasible solution for which stream i is serviced exactly $\mathrm{m}(\mathrm{i})$ times (some of which may be consecutive), and there exists an optimal such solution relative to the particular $\mathrm{m}(\mathrm{i})$ 's.

Proof. By Theorem 3, if $\left\{L_{i j}\right\}$ is a set satisfying

$$
\sum_{j=1}^{m(i)} L_{i j}=T_{i},
$$

where each $L_{i j} \geqslant d_{i}$, then $L_{i j}$ represents a feasible solution. By lemma $2, q_{i}$ is uniquely determined by the switching pattern. But the vector of switching times is a point of

$$
[0, T]^{\Sigma m(i)}
$$

a compact set. The conditions (given in Lemma 2) defining those switching patterns which correspond to feasible solutions are linear inequalities in the $L_{i j}$ 's, and so in the switching times themselves; thus the minimization is to take place over a closed subset of the compact set.

It will be shown in appendix B that $W(T)$ is continuous in the vector of switching times. Since this in turn ranges over a compact set, an optimal solution must exist.

The last result permits us to state explicit conditions for the existence of an optimal solution in the simplest case, namely for constant $a_{i}$.

Corollary 5. Suppose $\mathrm{a}_{\mathrm{i}}(\mathrm{t})=\mathrm{a}_{\mathrm{i}}$, a constant. If $\left\{\mathrm{L}_{\mathrm{i}}\right\}$ are the service interval lengths of a switching pattern such that

$$
\sum_{j=1}^{m(i)} L_{i j} \geqslant m(i) d_{i}+a_{i} T / C_{i}
$$

then the pattern is feasible. Furthermore, if numbers $\mathrm{T}_{\mathrm{i}}>0$ exist such that

$$
\sum_{i=1}^{N} T_{i}=T
$$

and

$$
\mathrm{T}_{\mathrm{i}} \geqslant \mathrm{m}(\mathrm{i}) \mathrm{d}_{\mathrm{i}}+\mathrm{a}_{\mathrm{i}} \mathrm{T} / \mathrm{C}_{\mathrm{i}}
$$

then an optimal feasible solution exists.

Proof. From Theorem 3 and Lemma 4.

Now let $\rho_{\mathrm{i}}=a_{i} / C_{i}$ and $d=\sum_{i=1}^{N} m(i) d_{i}$. Then we have the following.

THEOREM 6. For constant $\mathrm{a}_{\mathrm{i}}$, the following conditions are necessary and sufficient for the existence of a feasible solution with $\mathrm{m}(\mathrm{i})$ servicings of stream i which is optimal with respect to the given set $\{\mathrm{m}(\mathrm{i})\}$ :

$$
\begin{gathered}
\sum_{\mathrm{i}=1}^{\mathrm{N}} \rho_{\mathrm{i}}<1, \\
\mathrm{~T} \geqslant \frac{\mathrm{d}}{1-\sum_{\mathrm{i}=1}^{N} \rho_{\mathrm{i}}} .
\end{gathered}
$$

Proof. If $\left\{L_{i j}\right\}$ is feasible, by Lemma 5 it must satisfy (4). Summing (4) over all $i$, we get

$$
T \geqslant \sum_{i=1}^{N} m(i) d_{i}+T \sum_{i=1}^{N}\left(a_{i} / C_{i}\right)=d+T \sum_{i=1}^{N} \rho_{i},
$$


or equivalently

$$
T\left(1-\sum_{i=1}^{N} \rho_{i}\right) \geqslant d>0 \text {. }
$$

Since $T>0$, we have $1-\sum_{i=1}^{N} \rho_{i}>0$, proving (5), and also

$$
T \geqslant \frac{d}{1-\sum_{i=1}^{N} \rho_{i}},
$$

proving (6).

Now suppose (5) and (6) are satisfied. Then (7) is also satisfied. Thus it is possible to find $T_{i}>0$ such that

$$
T_{i} \geqslant m(i) d_{i}+a_{i} T / C_{i} .
$$

The result follows from Corollary 5 .

\section{Optimal Solutions for Constant Arrival Rates and Equal $m(i)$ 's}

This section will deal with the special case in which each $a_{i}(t)$ is a constant function, and the $m(i)$ are all equal to some common value $m$. This case was treated by Rangarajan and Oliver (see footnote 1), with the further provision that $m=1$, that is, that each stream was serviced exactly once.

A method of finding the optimum service period length for each stream was found (see footnote 1), and this is the result to be generalized here. It will be shown that an optimum (not necessarily the optimum) switching pattern is obtained for the case $m(i)=m, a_{i}$ constant, when the interval $[0, T]$ is broken up into $m$ subintervals of equal length, the switching pattern is optimized over the first interval $[0, T / m]$ by the methods of [1] with one servicing per stream, and this pattern is repeated cyclically for each of the other $m-1$ subintervals. In addition to this, an auxiliary result (see footnote 1) will be used to find the optimum value of $m$.

Lemma 7. Suppose that in the interval $[\mathrm{s}, \mathrm{t}]$ the function $\mathrm{f}(\mathrm{x})$ is either increasing at a rate $\mathrm{p}$ or decreasing at a rate $-\mathrm{q}$ almost everywhere. Suppose that $\mathrm{f}(\mathrm{s})=\mathrm{f}(\mathrm{t})=0$ anc' that $\mathrm{f}(\mathrm{x}) \geqslant 0$ for $\mathrm{x} \epsilon(\mathrm{s}, \mathrm{t})$. Suppose further that $\mathrm{f}$ increases during $\left(\mathrm{s}, \mathrm{s}+\mathrm{A}_{1}\right)$, decreases during $\left(\mathrm{s}+\mathrm{A}_{1}, \mathrm{~s}+\mathrm{A}_{1}+\mathrm{B}_{1}\right)$, etc., where each interval of increase of length $\mathrm{A}_{\mathrm{i}}$ is followed by an interval of decrease of length $\mathrm{B}_{\mathrm{i}}$ and each interval of decrease of length $\mathrm{B}_{\mathrm{i}}$ is followed by an interval of increase of length $\mathrm{A}_{\mathrm{i}+1}$, the last intervals being of length $\mathrm{A}_{\mathrm{n}}$ and $\mathrm{B}_{\mathrm{n}}$ respectively. Then

$$
\int_{s}^{t} f(x) d x \geqslant \frac{p(p+q)}{2 q} \sum_{i=1}^{n} A_{i}^{2}
$$

Proof. The proof is geometric in nature and is by induction on $n$. First, for $n=1$ the inequality reduces to

$$
\int_{s}^{t} f(x) d x \geqslant \frac{p(p+q)}{2 q} A_{1}^{2}
$$

Referring to figure 1, we see that $\int_{s}^{t} f(x) d x$ is the area of the triangle whose altitude is $A_{1} p$ and whose base is $A_{1}+B_{1}$, where

$$
B_{1}=A_{1} p / q
$$


Thus $\int_{s}^{t} f(x) d x=\frac{p(p+q)}{2 q} A_{1}^{2}$, proving the case $n=1$.

Assume that the lemma has been proved for $1,2, \ldots, n-1$. Looking at figure 2 , we see that

$$
\begin{aligned}
\int_{s}^{t} f(x) d x \geqslant \frac{p(p+q)}{2 q} \sum_{i=1}^{n-1} A_{i}^{2}+\text { Area } Y W V Z X & \geqslant \frac{p(p+q)}{2 q} \sum_{i=1}^{n-1} A_{i}^{2} \Delta X Y Z \\
& =\frac{p(p+q)}{2 q} \sum_{i=1}^{n-1} A_{i}^{2}+\frac{p(p+q)}{2 q} A_{n}^{2}=\frac{p(p+q)}{2 q} \sum_{i=1}^{n} A_{i}^{2} .
\end{aligned}
$$

We now state the following notations to be used during the rest of this section. $I_{i j}$ will denote the $j$ th interval of service for lane $i$ during $[0, T]$. Because of the cyclic character of the problem we also define

$$
I_{i k} \equiv I_{i j}
$$

where $1 \leqslant j \leqslant m$ and $k \equiv j(\bmod m)$. We denote the length of $I_{i j}$ by $L_{i j}$. The interval contained between $I_{i j}$ and $I_{i, j+1}$ (or the two intervals, if $j=m$ ) will be denoted by $J_{i j}$ and its length (respectively, the sum of their lengths) by $M_{i j}$.

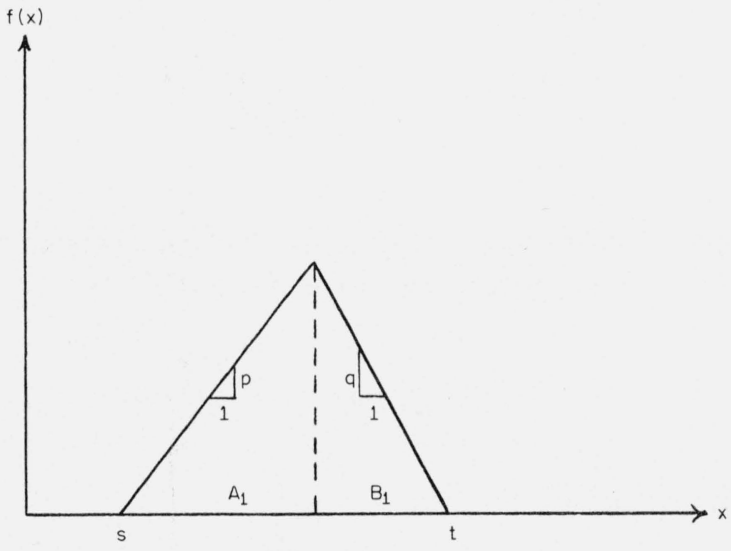

Figure 1.

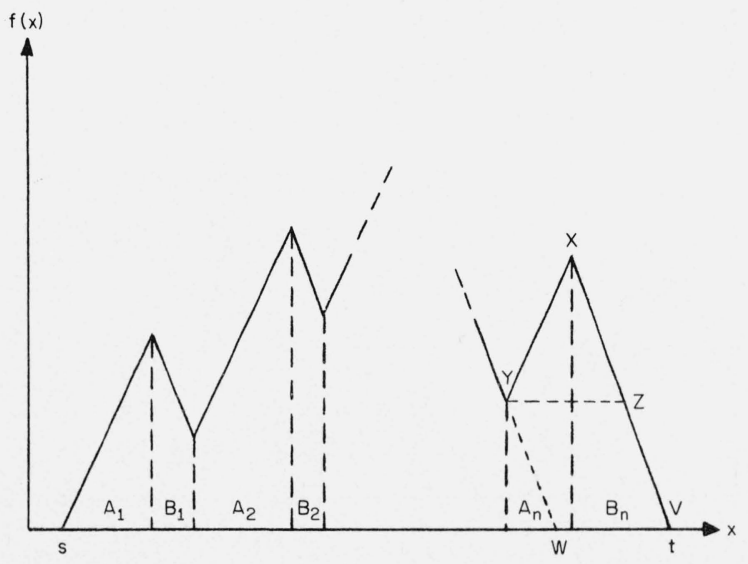

Figure 2. 
LEMMA 8. Let $\mathrm{S}$ be a feasible switching pattern for a problem with constant $\mathrm{a}_{\mathrm{i}}$. Then

$$
W_{i}(T) \geqslant \frac{a_{i} C_{i}}{2\left(C_{i}-a_{i}\right)} \sum_{j=1}^{m}\left(M_{i j}+d_{i}\right)^{2}
$$

Proof. By definition, $W_{i}$ is the integral of $Q_{i}$, which is always nonnegative. Thus $W_{i}$ may be thought of as the area under the curve representing $Q_{i}$. Since $C_{i}>a_{i}$ in order to satisfy feasibility, $Q_{i}$ is either rising at a rate $a_{i}$ (when $s_{i}=0$ ), falling at a rate $-\left(C_{i}-a_{i}\right.$ ) (when $s_{i}=C_{i}$ ), or constant at 0 (when $s_{i}=a_{i}$ ). We may represent this as in figure 3. It is also clear that the curve is rising only during the time that lane $i$ is not being serviced, plus the dead time, that is, during intervals of length $M_{i j}+d_{i}$.

Now let us group the $I_{i j}$ as follows. By condition (3), there exists $t_{0} \epsilon[0, T]$ such that $Q_{i}\left(t_{0}\right)=0$. Let $I_{i j}$ be the interval of service to lane $i$ to which $t_{0}$ belongs. (Obviously $t_{0}$ must lie in some interval of service.) Let $I_{i k}$ be the first interval after $I_{i j}$ during which $Q_{i}(t)$ is again 0 . Then the lengths of the intervals of nonservice to $i$ between $I_{i j}$ and $I_{i k}$, namely $J_{i j}, J_{i, j+1}, \ldots, J_{i, k-1}$, will be $M_{i j}$, $M_{i, j+1}, \ldots, M_{i, k-1}$. If we let $z_{i q}$ represent the right endpoint of any interval $I_{i q}$, then from Lemma 7 we have

$$
\int_{z_{i j}}^{z_{i k}} Q_{i}(t) d t \geqslant \frac{a_{i} C_{i}}{2\left(C_{i}-a_{i}\right)} \sum_{\nu=j}^{k-1}\left(M_{i \nu}+d_{i}\right)^{2} .
$$

Summing this inequality over all intervals in which $Q_{i}$ becomes 0 , we get

$$
W_{i}(T)=\int_{0}^{T} Q_{i}(t) d t \geqslant \frac{a_{i} C_{i}}{2\left(C_{i}-a_{i}\right)} \sum_{j=1}^{m}\left(M_{i j}+d_{i}\right)^{2}
$$

This proves the lemma.

THEOREM 9. Suppose that, for constant $\mathrm{a}_{\mathrm{i}},\left\{\mathrm{I}_{\mathrm{ij}}\right\}$ represents an optimal feasible solution, where $\mathrm{m}\left(\mathrm{i}=\mathrm{m}\right.$. Then there exists an optimal feasible solution $\left\{\overline{\mathrm{I}}_{\mathrm{ij}}\right\}$ with

$$
\bar{L}_{\mathrm{ij}}=(1 / \mathrm{m}) \sum_{k=1}^{\mathrm{m}} \mathrm{L}_{\mathrm{ik}}, \quad \mathrm{l} \leqslant \mathrm{i} \leqslant \mathrm{n},
$$

where $\overline{\mathrm{L}}_{\mathrm{ij}}$ is the length of interval $\overline{\mathrm{I}}_{\mathrm{ij}}$, $\mathrm{L}_{\mathrm{ij}}$ the length of $\mathrm{I}_{\mathrm{ij}}$.

Proof. Consider the solution defined by $\bar{L}_{i j}=(1 / m) \sum_{i=1}^{m} L_{i k}$, where $\bar{I}_{1,1}$ is the first interval and

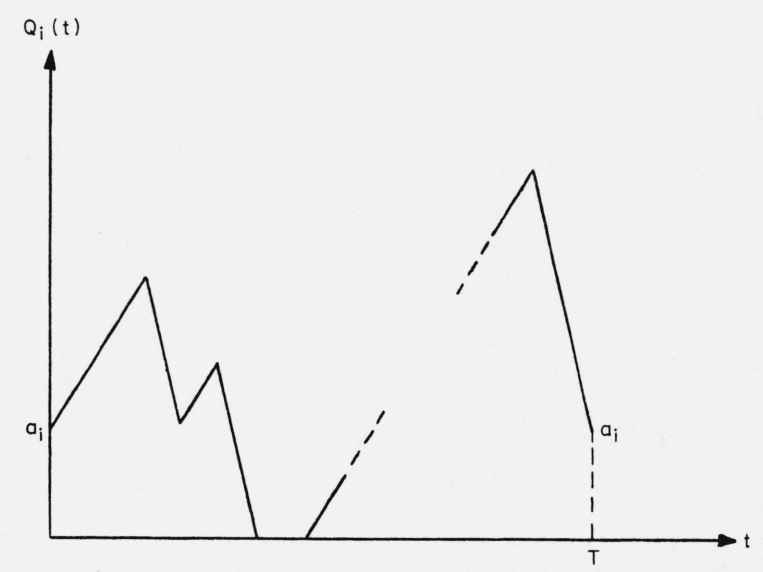

FigURE 3. 
where interval $\bar{I}_{i j}$ is followed by $\bar{I}_{i+1, j}$ for $i<n$, and interval $\bar{I}_{n j}$ is followed by $\bar{I}_{1, j+1}$. Then we have

$$
\bar{M}_{i j}=\bar{M}_{i}, \quad 1 \leqslant j \leqslant n .
$$

By lemma 8, the waiting time for the original solution satisfies

$$
W_{i}(T) \geqslant \frac{a_{i} C_{i}}{2\left(C_{i}-a_{i}\right)} \sum_{j=1}^{m}\left(M_{i j}+d_{i}\right)^{2},
$$

and the Cauchy-Schwartz inequality yields

$$
\sum_{j=1}^{m}\left(M_{i j}+d_{i}\right)^{2} \geqslant m\left(\bar{M}_{i j}+d_{i}\right)^{2}=\sum_{j=1}^{m}\left(\bar{M}_{i j}+d_{i}\right)^{2} .
$$

Thus it suffices to show that the new solution (which by Corollary 5 is feasible) obeys Lemma 8 with equality, i.e., that

$$
\bar{W}_{i}(T)=\int_{0}^{T} \bar{Q}_{i}(t) d t=\frac{a_{i} C_{i}}{2\left(C_{i}-a_{i}\right)} \sum_{j=1}^{m}\left(\bar{M}_{i j}+d_{i}\right)^{2} .
$$

From the proofs of Lemmas 7 and 8, it should be apparent that this is equivalent to proving that $\bar{Q}_{i}$ is reduced to 0 during each service interval $\bar{I}_{i j}$. The condition for this is

$$
\left(C_{i}-a_{i}\right)\left(\bar{L}_{i j}-d_{i}\right) \geqslant a_{i}\left(\bar{M}_{i j}+d_{i}\right)
$$

which will now be demonstrated.

By corollary 5 ,

$$
m \bar{L}_{i j}=\sum_{j=1}^{m} L_{i j} \geqslant m d_{i}+a_{i} T / C_{i}
$$

and so

$$
C_{i}\left(\bar{L}_{i j}-d_{i}\right) \geqslant a_{i}(T / m)=a_{i}\left(\bar{M}_{i j}+d_{i}+\left(\bar{L}_{i j}-d_{i}\right)\right),
$$

implying (8) as desired.

Note that Theorem 9 merely establishes the form of one optimal solution. It is clear that we could get a set of optimal solutions by permuting indices. Furthermore, it could probably be shown that these solutions are the only ones which are optimal, but this would involve solving a set of simultaneous linear equations relating the $M_{i j}$ and $L_{i j}$ and this is not considered here to be worth the trouble.

Also, nothing is said about the case where $m(i) \neq m(k)$. This seems to be a much harder case to analyze generally. However, if $N=2$ the above proof may be modified so that this problem is completely solved. For in that case it is obvious that $m(1)=m(2)$, since otherwise two servicings of some lane would be consecutive, giving a higher value for $W$ than that obtained by merging the two consecutive servicings into one. Also, $L_{i j}=M_{2, j-1}$ and $L_{2 j}=M_{i j}$. Thus the condition $M_{i j}=M_{i k}$ is equivalent to $L_{i j}=L_{i k}$.

Finally, we shall discuss the optimum value of $m$ to choose in the above problem, given $T$. Note that if $\left\{I_{i j}\right\}$ denotes an optimal solution on the interval $[0, T]$ as described above, where each lane is serviced once during the interval $[0, T / m]$ and this procedure is repeated $m$ times, then we have 


$$
m W(T / m)=W(T)
$$

This recalls the work of Rangarajan and Oliver (see footnote 1) in which the function to be minimized is $W(T) / T$ instead of $W(T)$. (Of course, for constant $T$, minimizing $W(T)$ is equivalent to minimizing $W(T) / T$, and the first part (see footnote 1) considers the problem in this way.) We show how the results can be applied to the above problem.

Suppose that $T^{\prime}$ is a variable which is allowed to take on only the discrete set of values $T / \mathrm{m}$, $m$ an integer, in the problem where each lane is serviced exactly once during the interval $\left[0, T^{\prime}\right]$. If $W\left(T^{\prime}\right) / T^{\prime}$ attains its minimum value on this discrete set at the point $T^{\prime}=T_{0}^{\prime}=T / m_{0}$, then $W(T)$ in the original problem attains its minimum value over all $m$ for $m=m_{0}$. For we have

$$
\frac{W\left(T / m_{0}\right)}{T / m_{0}} \leqslant \frac{W(T / m)}{T / m}
$$

for $m \neq m_{0}$; or,

$$
m_{0} W\left(T / m_{0}\right) \leqslant m W(T / m) .
$$

But by the above remarks, $m W(T / m)=W(T)$ where $W(T)$ is calculated for $m$ cycles of service in the interval $[0, T]$, and similarly for $m_{0} W\left(T / m_{0}\right)$.

Thus it is clear that finding $m_{0}$ such that $W(T)$, optimized over all switching patterns, attains its least value when each stream is serviced $m_{0}$ times, is equivalent to finding $m_{0}$ such that $W\left(T / m_{0}\right) /$ $\left(T / m_{0}\right)$ is a minimum for the optimized solution of the one-cycle case. Now a method is given (see footnote 1) to optimize $W\left(T^{\prime}\right) / T^{\prime}$ when $T^{\prime}$ is a continuous variable, and it is shown that $W\left(T^{\prime}\right) / T^{\prime}$ is monotone decreasing to the left of the optimum, $T^{\prime}=T_{0}^{\prime}$, and monotone increasing to the right. Thus if $n$ is an integer such that

$$
T /(n+1) \leqslant T_{0}^{\prime} \leqslant T / n
$$

it is clear that the optimum value $m_{0}$ sought above must be either $n$ or $n+1$. In order to determine which of these values is optimum, it is only necessary to substitute in the expression for the optimized value of $W\left(T^{\prime}\right) / T^{\prime}$, also given (see footnote 1). Thus $m_{0}$ is easily determined.

\section{Appendix A: Uniqueness of $Q_{i}$}

We show here that the $s_{i}$, and hence the $Q_{i}$, are well defined by the definition given in the text. First, it is clear that $s_{i}(t)=0$ when $t$ is not in some interval of the form $\left(x_{i j}+d_{i}, y_{i j}\right)$. Let us divide each interval $\left(x_{i j}+d_{i}, y_{i j}\right)$ into subintervals in which either $a_{i}(t)>C_{i}$ or $a_{i}(t) \leqslant C_{i}$ for all $t$ in the subinterval. It is clear that there will be a finite number of such subintervals, since $a_{i}(t)$ crosses the value $C_{i}$ at most a finite number of times.

In the subintervals where $a_{i}(t)>C_{i}$, it is clear that $a_{i}(t)-s_{i}(t)>0$, so that $Q_{i}(t)>0$ and hence we must have $s_{i}(t)=C_{i}$.

Now assume that $[b, c]$ is a subinterval such that $a_{i}(t) \leqslant C_{i}$ for $t \epsilon[b, c]$, and that $Q_{i}$ has been defined for $t \epsilon[0, b]$. Let

$$
\bar{Q}_{i}(t)=Q_{i}(b)+\int_{b}^{t}\left(a_{i}(\tau)-C_{i}\right) d \tau
$$

and $Q_{i}(t)=\max \left(\bar{Q}_{i}(t), 0\right)$, for $t \epsilon[b, c]$. We will show that this gives the only possible value for $Q_{i}$. 
For if $\bar{Q}_{i}(t)>0$ for some $t \epsilon[b, c]$, then since $Q_{i}$ is changing in $[b, t]$ at a rate at least as great as $a_{i}(\tau)-C_{i}$, it is clear that $Q_{i} \geqslant \bar{Q}_{i}$. Thus, $s_{i}(t)=C_{i}$ for such $t$ and so $Q_{i}=\bar{Q}_{i}$. Now if $\bar{Q}_{i}(t) \leqslant 0$ for some $t \epsilon[b, c]$, since $\bar{Q}_{i}$ is continuous we can find a first point $\tau$ in $[b, c]$ where $\bar{Q}_{i}(\tau)=0$. By the above argument, $Q_{i}(\tau)=0$ also. Since $s_{i}(t) \geqslant a_{i}(t)$ in the interval $[b, c]$, we must then have that $Q_{i}(t) \leqslant 0$ for all $t \epsilon[\tau, c]$, by the definition of $Q_{i}$, and so $Q_{i}(t)=0$, satisfying the above assertion.

\section{Appendix B: Continuity of the Objective Function}

We next show that the value $W(T)$ associated with a feasible solution defined by a given switching pattern

$$
\left\{\left[x_{i j}, y_{i j}\right]\right\}
$$

is uniformly continuous in the $x_{i j}$ and $y_{i j}$. Let $\left\{\left[x_{i j}, y_{i j}\right]\right\}$ and $\left\{\left[x_{i j}^{\prime}, y_{i j}^{\prime}\right]\right\}$. be two switching patterns such that, for some stream $r$, the service intervals for stream $r$ are exactly the same in both patterns except for one interval endpoint, say $x_{r p}$. Let $Q_{r}$ and $Q_{r}^{\prime}$ be the respective queue-size functions for stream $r$ associated with $\left\{\left[x_{i j}, y_{i j}\right]\right\}$ and $\left\{\left[x_{i j}^{\prime}, y_{i j}^{\prime}\right]\right\}$. Suppose that $x_{r p}^{\prime}<x_{r p}$, so that stream $r$ is serviced more by the second pattern. Let $q_{r}=Q_{r}(0)$ and $q_{r}^{\prime}=Q_{r}^{\prime}(0)$.

Construct a new queue-size function $\bar{Q}_{r}$ as the (possibly not feasible) function associated with initial size $q_{r}$ and switching pattern $\left\{\left[x_{i j}^{\prime}, y_{i j}^{\prime}\right]\right\}$. It is clear, by the previous section, that

$$
\bar{Q}_{r}(t)=Q_{r}(t)
$$

for $t \epsilon\left[0, x_{r p}^{\prime}\right]$. Also, since

$$
s_{r}(t) \geqslant \bar{s}_{r}(t)
$$

for $t \epsilon\left[x_{r p}^{\prime}, x_{r p}\right]$, where $s_{r}$ and $\bar{s}_{r}$ are the service-rate functions of the two patterns, we have

$$
\bar{Q}_{r}(t) \leqslant Q_{r}(t)
$$

for $t \epsilon\left[x_{r p}^{\prime}, x_{r p}\right]$, and hence $\bar{Q}_{r}\left(x_{r p}\right) \leqslant Q_{r}\left(x_{r p}\right)$. Since the two patterns are the same for the rest of the interval, $\left[x_{r p}, T\right]$, we have by lemma 1 that

$$
\bar{Q}_{r}(t) \leqslant Q_{r}(t)
$$

for $t \epsilon\left[x_{r p}, T\right]$.

Let $\overline{\bar{Q}}_{r}$ be the queue-size function defined by

$$
\overline{\bar{Q}}_{r}(0)=\bar{q}_{r}=\bar{Q}_{r}(T)
$$

and having a switching pattern $\left\{\left[x_{i j}^{\prime}, y_{i j}^{\prime}\right]\right\}$. Since $Q_{r}(t)$ represents a feasible solution, there exists $\tau \epsilon[0, T]$ such that $Q_{r}(\tau)=0$. But then

$$
\bar{Q}_{r}(\tau) \leqslant Q_{r}(\tau)=0
$$

implying $\bar{Q}_{r}(\tau)=0$. Now $\overline{\bar{Q}}_{r}$ has the same switching pattern as $\bar{Q}_{r}$, and

$$
\bar{q}_{r}=\bar{Q}_{r}(T) \leqslant Q_{r}(T)=q_{r},
$$

so that $\overline{\bar{Q}}_{r}(t) \leqslant \bar{Q}_{r}(t)$ for all $t \epsilon[0, T]$, and hence

$$
\overline{\bar{Q}}_{r}(\tau) \leqslant \bar{Q}_{r}(\tau)=0,
$$


implying $\overline{\bar{Q}}_{r}(\tau)=0$. Since $\overline{\bar{Q}}_{r}$ and $\bar{Q}_{r}$ have the same switching pattern we may then conclude that

$$
\overline{\bar{Q}}_{r}(t)=\bar{Q}_{r}(t)
$$

for $t \epsilon[\tau, T]$, and so

$$
\overline{\bar{Q}}_{r}(T)=\bar{Q}_{r}(T)=\bar{q}_{r}
$$

Thus $\overline{\bar{Q}}_{r}$ is feasible and so $Q_{r}^{\prime}=\overline{\bar{Q}}_{r}$, by lemma 2 .

But $\left\{\left[x_{i j}, y_{i j}\right]\right\}$ and $\left\{\left[x_{i j}^{\prime}, y_{i j}\right]\right\}$ differ only on the portion $\left(x_{r p}^{\prime}, x_{r p}\right)$, as far as stream $r$ is concerned, and so $Q_{r}$ and $Q_{r}^{\prime}$ may differ by at most

$$
C_{r}\left(x_{r p}-x_{r p}^{\prime}\right)
$$

by the above and by Lemma 1 .

Now consider any two feasible switching patterns $\left\{\left[x_{i j}, y_{i j}\right]\right\}$ and $\left\{\left[x_{i j}^{\prime}, y_{i j}^{\prime}\right]\right\}$, and let $\delta_{i}$ be the shortest distance between successive intervals of service to the $i$ th stream in either pattern. That is,

$$
\delta_{i}=\min \left\{\min _{j}\left(x_{i, j+1}-y_{i j}\right), \min _{j}\left(x_{i, j+1}^{\prime}-y_{i j}^{\prime}\right)\right\}
$$

If we have $\left|x_{i j}-x_{i j}^{\prime}\right|<\delta_{i} / 3$ and $\left|y_{i j}-y_{i j}^{\prime}\right|<\delta_{i} / 3$ for all $j$, then

$$
\left|Q_{i}(t)-Q_{i}^{\prime}(t)\right| \leqslant C_{i} \sum_{j}\left(\left|x_{i j}-x_{i j}^{\prime}\right|+\left|y_{i j}-y_{i j}^{\prime}\right|\right)
$$

For we may find a sequence of feasible switching patterns connecting $\left\{\left[x_{i j}, y_{i j}\right]\right\}$ and $\left[\left\{x_{i j}^{\prime}, y_{i j}^{\prime}\right]\right\}$ such that each two adjacent patterns of the sequence differ in only one coordinate. This is done by first changing the $x_{i j}$ to $x_{i j}^{\prime}$ and the $y_{i j}$ to $y_{i j}^{\prime}$ so that at each step the interval length $L_{i j}$ is increased. When no more such changes are possible, the remaining $x_{i j}$ are changed to $x_{i j}^{\prime}$ and the remaining $y_{i j}$ to $y_{i j}^{\prime}$. Since the sum of interval lengths is not decreased below the smaller of the sum of interval lengths for the primed and the unprimed patterns during this process, and since no intervals overlap during the process (because changes in endpoints are smaller than $\delta_{i} / 3$ ), all switching patterns in the sequence are feasible.

Finally, for any two switching patterns $\left\{\left[x_{i j}, y_{i j}\right]\right\}$ and $\left\{\left[x_{i j}^{\prime}, y_{i j}^{\prime}\right]\right\}$ we assert that

$$
\left|Q_{i}(t)-Q_{i}^{\prime}(t)\right| \leqslant C_{i} \sum_{j}\left(\left|x_{i j}-x_{i j}^{\prime}\right|+\left|y_{i j}-y_{i j}^{\prime}\right|\right)
$$

For it is possible to find a set of intermediate switching patterns

$$
\left\{\left[x_{i j}^{0}, y_{i j}^{0}\right]\right\},\left\{\left[x_{i j}^{1}, y_{i j}^{1}\right]\right\}, \ldots .\left\{\left[x_{i j}^{m}, y_{i j}^{m}\right]\right\}
$$

connecting $\left\{\left[x_{i j}, y_{i j}\right]\right\}$ and $\left\{\left[x_{i j}^{\prime}, y_{i j}^{\prime}\right]\right\}$ whose interval separations are all at least as great as the smallest interval separation, $\delta_{i}$, of the given pair of patterns. This is done merely by defining

$$
x_{i j}^{k}=\frac{k}{m} x_{i j}+\frac{m-k}{m} x_{i j}^{\prime},
$$




$$
y_{i j}^{k}=\frac{k}{m} y_{i j}+\frac{m-k}{m} y_{i j}^{\prime}
$$

If $m$ is chosen large enough, then we also have that

$$
\left|x_{i j}^{k}-x_{i j}^{k+1}\right|<\delta_{i} / 3, \quad\left|y_{i j}^{k}-y_{i j}^{k+1}\right|<\delta_{i} / 3
$$

so that the above inequality for $\left|Q_{i}-Q_{i}^{\prime}\right|$ follows easily.

This proves uniform continuity of the $Q_{i}$ in the switching pattern. A proof of the continuity of $W(T)$ in the switching pattern follows from elementary calculus.

(Paper 72B3-271) 